\title{
On complete totally umbilical and maximal space-like surfaces in pseudo-Riemannian manifolds
}

\author{
Irina I. Tsyganok and Sergey E. Stepanov
}




\title{
ON COMPLETE TOTALLY UMBILICAL AND MAXIMAL SPACE-LIKE SURFACES IN PSEUDO-RIEMANNIAN MANIFOLDS
}

\author{
IRINA I. TSYGANOK AND SERGEY E. STEPANOV
}

\begin{abstract}
Space-like surfaces with special second fundamental forms have been an important tool in the study of pseudo-Riemannian manifolds. This paper focuses on adapting some theorems of Riemannian geometry in the large to the study of geometry of space-like surfaces of pseudo-Riemannian manifolds, specifically, various comparison theorems that use sectional and Ricci curvatures. The purpose of this paper is to establish a global geometry of complete spacelike totally umbilical and maximal surfaces in pseudo-Riemannian manifolds.
\end{abstract}

2000 Mathematics Subject Classification: 53C50; 83C75

Keywords: pseudo-Riemannian manifold, Lorentzian manifold, de Sitter space, space-like totally umbilical and maximal hypersurfaces

\section{DEFinitions AND NOTATIONS}

First, we set up our terminology and notations (see $[2,4])$. Let $(M, g)$ be an $n$ dimensional connected pseudo-Riemannian manifold (also called a semi-Riemannian manifold) of class $C^{\infty}$ whose metric $g$ has the signature $(p, q)$ for $p+q=n$ and $M^{\prime}$ an $n^{\prime}$-dimensional differentiable manifold of class $C^{\infty}$ imbedded in $(M, g)$ with an imbedding map $f: M^{\prime} \rightarrow M$. We call the image $f\left(M^{\prime}\right)$ a surface (also called a submanifold) in $(M, g)$ and identify it with the manifold $M^{\prime}$.

The differential $d f$ of the imbedding map $f: M^{\prime} \rightarrow M$ will denote by $f_{*}$, so that a vector field $X^{\prime}$ in $T M^{\prime}$ corresponds to a vector field $f_{*} X^{\prime}$ in $T M$.

We denote by $g^{\prime}=g\left(f_{*}, f_{*}\right)$ the metric tensor $g^{\prime}=f^{*} g$ induced in $M^{\prime}$ from $g$ by $f$, where $f^{*}$ is the mapping conjugate of $f_{*}$. Then, the surface $M^{\prime}$ is called space-like if the metric tensor $g^{\prime}$ is positive definite and hence $n^{\prime} \leq q$. In this case, $\left(M^{\prime}, g^{\prime}\right)$ is a Riemannian manifold and for each of its points $x$, there exists a normal subspace of a tangent space $T_{x} M$ such that the following orthogonal decomposition $T_{x} M^{\prime}=\left(T_{x} M^{\prime}\right)^{\perp} \oplus T_{x} M^{\prime}$ is true.

We denote by $\nabla^{\prime}$ the covariant differential operator corresponding to the Riemannian metric $g^{\prime}$. Then, the second fundamental form $Q$ of the surface $\left(M^{\prime}, g^{\prime}\right)$ is defined by the formula

$$
Q\left(X^{\prime}, Y^{\prime}\right):=\left(\nabla^{\prime} X^{\prime} f_{*}\right) Y^{\prime}=\nabla_{f_{*} X^{\prime}} f_{*} Y^{\prime}-f_{*}\left(\nabla^{\prime} X^{\prime} Y^{\prime}\right)
$$


for any $X^{\prime}, Y^{\prime} \in C^{\infty} T M^{\prime}$. The Gauss curvature equation for $\left(M^{\prime}, g^{\prime}\right)$ has the form

$$
\begin{aligned}
R^{\prime}\left(X^{\prime}, Y^{\prime}, V^{\prime}, W^{\prime}\right) & =R\left(f_{*} X^{\prime}, f_{*} Y^{\prime}, f_{*} V^{\prime}, f_{*} W^{\prime}\right) \\
+ & g\left(Q\left(X^{\prime}, W^{\prime}\right), Q\left(Y^{\prime}, V^{\prime}\right)\right)-g\left(Q\left(Y^{\prime}, W^{\prime}\right), Q\left(X^{\prime}, V^{\prime}\right)\right)
\end{aligned}
$$

for all $X^{\prime}, Y^{\prime}, V^{\prime}, W^{\prime} \in C^{\infty} T M^{\prime}$, where $R^{\prime}$ is the curvature tensor of $\left(M^{\prime}, g^{\prime}\right)$. In particular, if $(M, g)$ is a pseudo-Riemannian manifold of constant curvature $C$, then (1.1) can be rewritten in the form

$$
\begin{aligned}
R^{\prime}\left(X^{\prime}, Y^{\prime}, V^{\prime}, W^{\prime}\right)= & C \cdot\left(g^{\prime}\left(X^{\prime}, W^{\prime}\right) g^{\prime}\left(Y^{\prime}, V^{\prime}\right)-g^{\prime}\left(Y^{\prime}, W^{\prime}\right) g^{\prime}\left(X^{\prime}, V^{\prime}\right)\right)+ \\
& g\left(Q\left(X^{\prime}, W^{\prime}\right), Q\left(Y^{\prime}, V^{\prime}\right)\right)-g\left(Q\left(Y^{\prime}, W^{\prime}\right), Q\left(X^{\prime}, V^{\prime}\right)\right) .
\end{aligned}
$$

A surface $\left(M^{\prime}, g^{\prime}\right)$ is said to be totally umbilical in $(M, g)$ if its second fundamental form satisfies $Q=g^{\prime} \cdot H$ where $H$ is the mean curvature vector defined by the formula $n \cdot H=$ trace $_{g^{\prime}} Q$. In this case, (1.1) can be rewritten as the following equations

$$
\begin{aligned}
& R^{\prime}\left(X^{\prime}, Y^{\prime}, V^{\prime}, W^{\prime}\right)=R\left(f_{*} X^{\prime}, f_{*} Y^{\prime}, f_{*} V^{\prime}, f_{*} W^{\prime}\right) \\
& \quad+g(H, H) \cdot\left(g^{\prime}\left(X^{\prime}, W^{\prime}\right) g^{\prime}\left(Y^{\prime}, V^{\prime}\right)-g^{\prime}\left(Y^{\prime}, W^{\prime}\right) g^{\prime}\left(X^{\prime}, V^{\prime}\right)\right)
\end{aligned}
$$

for all $X^{\prime}, Y^{\prime}, V^{\prime}, W^{\prime} \in C^{\infty} T M^{\prime}$. In addition, if $(M, g)$ is a manifold of constant curvature $C$, then from (1.2) and (1.3), we obtain

$$
\begin{aligned}
R^{\prime}\left(X^{\prime}, Y^{\prime}, V^{\prime}, W^{\prime}\right)=(C+g(H, H)) \cdot\left(g^{\prime}\left(X^{\prime}, W^{\prime}\right) g^{\prime}\left(Y^{\prime}, V^{\prime}\right)\right. \\
\\
\left.-g^{\prime}\left(Y^{\prime}, W^{\prime}\right) g^{\prime}\left(X^{\prime}, V^{\prime}\right)\right)
\end{aligned}
$$

and hence, $\left(M^{\prime}, g^{\prime}\right)$ is a manifold of constant curvature $C^{\prime}=C+g(H, H)$.

On the other hand, from (1.3), we obtain

$$
\sec ^{\prime}(x, \pi)=\sec (x, \pi)+g\left(H_{x}, H_{x}\right)
$$

where

$$
\begin{aligned}
\sec ^{\prime}(x, \pi) & =\sec ^{\prime}\left(X_{x}^{\prime} \wedge Y_{x}^{\prime}\right) \\
& :=-\frac{R^{\prime}\left(X_{x}^{\prime}, Y_{x}^{\prime}, X_{x}^{\prime}, Y_{x}^{\prime}\right)}{g^{\prime}\left(X_{x}^{\prime}, X_{x}^{\prime}\right) g^{\prime}\left(Y_{x}^{\prime}, Y_{x}^{\prime}\right)-g^{\prime}\left(X_{x}^{\prime}, Y_{x}^{\prime}\right)^{2}}
\end{aligned}
$$

and

$$
\begin{aligned}
\sec (x, \pi) & =\sec \left(X_{x}^{\prime} \wedge Y_{x}^{\prime}\right) \\
& :=-\frac{R\left(f_{*} X_{x}^{\prime}, f_{*} Y_{x}^{\prime}, f_{*} X_{x}^{\prime}, f_{*} Y_{x}^{\prime}\right)}{g\left(f_{*} X_{x}^{\prime}, f_{*} X_{x}^{\prime}\right) g\left(f_{*} Y_{x}^{\prime}, f_{*} Y_{x}^{\prime}\right)-g\left(f_{*} X_{x}^{\prime}, f_{*} Y_{x}^{\prime}\right)^{2}}
\end{aligned}
$$

are space-like sectional curvatures of $\left(M^{\prime}, g^{\prime}\right)$ and $(M, g)$ at $x \in M^{\prime}$ with respect to the space-like nondegenerated plane $\pi=\operatorname{span}\left\{X_{x}^{\prime}, Y_{x}^{\prime}\right\} \subset T_{x} M^{\prime}$. 
In particular, let $\left(M^{\prime}, g^{\prime}\right)$ be an $n$-dimensional space-like totally umbilical hypersurface (also called a hypersubmanifold) in a Lorentzian manifold $(M, g)$. It means that $(M, g)$ is a $(n+1)$-dimensional pseudo-Riemannian manifold $(M, g)$ with metric $g$ of Lorentzian signature $(-,+, \ldots,+)$. In this case, we can rewrite the equality (1.5) in the following form

$$
\sec ^{\prime}(x, \pi)=\sec (x, \pi)-\left|H^{2}\right|
$$

where $\left|H^{2}\right|=|g(H, H)|$ is a mean curvature of $\left(M^{\prime}, g^{\prime}\right)$.

Next, a surface $\left(M^{\prime}, g^{\prime}\right)$ is said to be maximal (in contrast to the Riemannian case where it is called the minimal surface) if its mean curvature vector $H=0$. In particular, if $\left(M^{\prime}, g^{\prime}\right)$ is an $n$-dimensional space-like maximal hypersurface in a Lorentzian manifold $(M, g)$, the following equation can be obtained from (1.1)

$$
\operatorname{Ric}^{\prime}\left(Y^{\prime}, V^{\prime}\right)=\operatorname{Ric}\left(f_{*} Y^{\prime}, f_{*} V^{\prime}\right)+R\left(\mathcal{N}, f_{*} Y^{\prime}, f_{*} V^{\prime}, \mathcal{N}\right)+g^{\prime}\left(\mathcal{A} Y^{\prime}, \mathcal{A} V^{\prime}\right)
$$

where Ric ${ }^{\prime}$ and Ric are Ricci tensors of $\left(M^{\prime}, g^{\prime}\right)$ and $(M, g)$, respectively. Moreover, $\mathcal{N}$ is the (globally defined) unitary time-like normal vector field on $M^{\prime}$ and $\mathcal{A}$ is the shape operator of $\left(M^{\prime}, g^{\prime}\right)$ in $(M, g)$ with respect to $\mathcal{N}$ such that $Q\left(Y^{\prime}, V^{\prime}\right)=$ $-g^{\prime}\left(\mathcal{A} Y^{\prime}, V^{\prime}\right) \mathcal{N}$. In particular, from (1.7), we obtain

$$
\operatorname{Ric}^{\prime}\left(V_{x}^{\prime}\right)=\operatorname{Ric}\left(f_{*} V_{x}^{\prime}\right)-\sec (x, \pi)+\left\|\mathcal{A} V^{\prime}\right\|^{2} \cdot\left\|V_{x}^{\prime}\right\|^{-2}
$$

where $\operatorname{Ric}^{\prime}\left(V_{x}^{\prime}\right)=\operatorname{Ric}^{\prime}\left(V_{x}^{\prime}, V_{x}^{\prime}\right) \cdot\left\|V_{x}\right\|^{-2}$ and $\operatorname{Ric}\left(f_{*} V_{x}^{\prime}\right)=\operatorname{Ric}^{\prime}\left(f_{*} V_{x}^{\prime}, f_{*} V_{x}^{\prime}\right)$. $\left\|V_{x}\right\|^{-2}$ are Ricci curvatures at $x \in M^{\prime}$ with respect to the nonzero vector $V_{x}^{\prime} \in$ $T_{x} M^{\prime}$,

$$
\left\|\mathcal{A} V^{\prime}\right\|^{2}=g^{\prime}\left(\mathcal{A} V_{x}^{\prime}, \mathcal{A} V_{x}^{\prime}\right)
$$

and

$$
\left\|V_{x}\right\|^{2}=g^{\prime}\left(V_{x}^{\prime}, V_{x}^{\prime}\right)=g\left(f_{*} V_{x}^{\prime}, f_{*} V_{x}^{\prime}\right) .
$$

Moreover, here, $\sec (x, \pi):=\sec \left(\mathcal{N}_{x} \wedge f_{*} V_{x}^{\prime}\right)$ is a time-like sectional curvature of the time-like plane $\pi=\operatorname{span}\left\{\mathcal{N}_{x}, f_{*} V_{x}^{\prime}\right\}$ and $V_{x}^{\prime} \in T_{x} M^{\prime}$ is a nonzero vector. Next, along with (1.8) from (1.7), we obtain the equation

$$
s=s^{\prime}+2 \operatorname{Ric}(\mathcal{N}, \mathcal{N})+\operatorname{trace}\left(\mathcal{A}^{2}\right)
$$

where $s^{\prime}$ and $s$ are scalar curvatures of $\left(M^{\prime}, g^{\prime}\right)$ and $(M, g)$, respectively.

Finally, $\left(M^{\prime}, g^{\prime}\right)$ is said to be totally geodesic if its second fundamental form $Q$ vanishes identically. Obviously, such a surface is totally umbilical and maximal at the same time. In this paper, we exclude such surfaces from consideration.

\section{TOTALLY UMBILICAL SURFACES IN PSEUDO-RIEMANNIAN MANIFOLDS}

Let $\left(M^{\prime}, g^{\prime}\right)$ be a $n$-dimensional complete space-like totally umbilical surface in a pseudo-Riemannian manifold $(M, g)$. In this case, we have 
Theorem 2.1. Let $\left(M^{\prime}, g^{\prime}\right)$ be a complete space-like totally umbilical surface in a pseudo-Riemannian manifold $(M, g)$.

(1) If the mean curvature vector $H$ of $\left(M^{\prime}, g^{\prime}\right)$ and the sectional curvature sec of $(M, g)$ satisfy the inequality

$$
\sec (\pi) \leq-g(H, H)
$$

for all plane sections $\pi$ of $T M^{\prime}$, then $\left(M^{\prime}, g^{\prime}\right)$ is diffeomorphic to $\mathbb{R}^{n}$.

(2) If there exists a constant $\delta>0$ such that the mean curvature vector $H$ of $\left(M^{\prime}, g^{\prime}\right)$ and the sectional curvature sec of $(M, g)$ satisfy the inequality

$$
\sec (\pi) \geq-g(H, H)+\delta
$$

for all plane sections $\pi$ of $T M^{\prime}$, then $\left(M^{\prime}, g^{\prime}\right)$ is compact, its diameter satisfies the inequality diam $M^{\prime} \leq \pi / \sqrt{\delta}$ and the first fundamental group is finite.

Proof. Firstly, we recall that Mayer's theorem states (see [5, pp. 212-213]) that a complete Riemannian manifold $\left(M^{\prime}, g^{\prime}\right)$ is compact if there exists a positive constant $\delta$ such that $\sec ^{\prime}(x, \pi) \geq \delta>0$ for an arbitrary plane $\pi=\operatorname{span}\left\{X_{x}^{\prime}, Y_{x}^{\prime}\right\}$ at each point $x \in M^{\prime}$. Moreover, its diameter satisfies the inequality diam $M^{\prime} \leq \pi / \sqrt{\delta}$ and the first fundamental group is finite.

Secondly, by (1.3), the inequality $\sec ^{\prime}(x, \pi) \geq \delta>0$ can be rewritten in the form

$$
\sec (x, \pi) \geq-g\left(H_{x}, H_{x}\right)+\delta .
$$

Thirdly, if a complete Riemannian manifold $\left(M^{\prime}, g^{\prime}\right)$ has nonpositive sectional curvature at all plane sections, then $\left(M^{\prime}, g^{\prime}\right)$ is diffeomorphic to $\mathbb{R}^{n}$ (see $[5$, p. 201]). After these remarks, the theorem statements become obvious.

Next, from Theorem 2.1 and (1.4), we conclude that if $(M, g)$ is a manifold of constant curvature $C$ such that $C+g(H, H)>0$, then its complete space-like totally umbilical surface $\left(M^{\prime}, g^{\prime}\right)$ is compact. In addition, we recall that any compact, simply-connected Riemannian manifold $\left(M^{\prime}, g^{\prime}\right)$ with constant sectional curvature $C^{\prime}>0$ is necessarily isometric to a Euclidian sphere $\mathbb{S}^{n}$ of radius $\left(C^{\prime}\right)^{-1 / 2}$, equipped with its standard metric (see [5, p. 217-218]). Thus, using Theorem 2.1 and the result above, we can formulate the following corollary.

Corollary 2.1. Let $\left(M^{\prime}, g^{\prime}\right)$ be a complete space-like totally umbilical surface in a pseudo-Riemannian manifold $(M, g)$ of constant curvature $C$. If the mean curvature vector $H$ of $\left(M^{\prime}, g^{\prime}\right)$ satisfies the inequality

$$
g(H, H)+C>0,
$$

then $\left(M^{\prime}, g^{\prime}\right)$ is compact and its first fundamental group is finite. Further, suppose that $\left(M^{\prime}, g^{\prime}\right)$ is simply-connected, then $\left(M^{\prime}, g^{\prime}\right)$ is isometric to a Euclidian sphere $\mathbb{S}^{n}$ of radius $(g(H, H)+C)^{-1 / 2}$. 
Now, we suppose that $\left(M^{\prime}, g^{\prime}\right)$ is an $n$-dimensional space-like totally umbilical hypersurface in a Lorentzian manifold $(M, g)$. In this case, from the inequality $\sec (x, \pi) \leq\left|H_{x}{ }^{2}\right|$, we obtain $\sec ^{\prime}(x, \pi) \leq 0$ for an arbitrary plane $\pi \subset T_{x} M^{\prime}$ at each point $x \in M^{\prime}$. It follows that $\left(M^{\prime}, g^{\prime}\right)$ is diffeomorphic to $\mathbb{R}^{n}$ (see [5, p. 201]).

On the other hand, if the inequality $\sec (x, \pi)>\left|H_{x}{ }^{2}\right|+\delta$ holds for some positive constant $\delta$, then from (1.5), we obtain $\sec ^{\prime}(x, \pi) \geq \delta>0$. Therefore, as a consequence of Mayer's theorem (see [5, pp. 212-213]), we have the following result.

Corollary 2.2. Let $\left(M^{\prime}, g^{\prime}\right)$ be a complete space-like totally umbilical hypersurface in a Lorentzian manifold $(M, g)$.

(1) If the mean curvature $\left|H^{2}\right|$ of $\left(M^{\prime}, g^{\prime}\right)$ and the sectional curvature sec of $(M, g)$ satisfy the inequality

$$
\sec (\pi) \leq\left|H^{2}\right|
$$

for all plane sections $\pi$ of $T M^{\prime}$, then $\left(M^{\prime}, g^{\prime}\right)$ is diffeomorphic to $\mathbb{R}^{n}$.

(2) If there exists a positive constant $\delta$ such that the mean curvature $\left|H^{2}\right|$ of $\left(M^{\prime}, g^{\prime}\right)$ and the sectional curvature $\sec$ of $(M, g)$ satisfy the inequality

$$
\sec (\pi) \geq\left|H^{2}\right|+\delta
$$

for all plane sections $\pi$ of $T M^{\prime}$, then $\left(M^{\prime}, g^{\prime}\right)$ is compact, its diameter satisfies the inequality

$$
\operatorname{diam} M^{\prime} \leq \frac{\pi}{\sqrt{\delta}}
$$

and the first fundamental group is finite.

We define a de Sitter space $\mathbb{S}_{1}^{n+1}(C)$ as a Lorentzian manifold $(M, g)$ with positive constant sectional curvature $C$ and recall that any space-like compact hypersurface $\left(M^{\prime}, g^{\prime}\right)$ is a de Sitter space $\mathbb{S}_{1}^{n+1}(C)$ diffeomorphic to a Euclidian sphere $\mathbb{S}^{n}$. In particular, compact totally umbilical space-like hypersurfaces in $\mathbb{\$}_{1}^{n+1}(C)$ are round $n$-spheres (see $[1,8])$. Now, we can formulate the following corollary.

Corollary 2.3. Let $\left(M^{\prime}, g^{\prime}\right)$ be a complete space-like totally umbilical hypersurface in $\mathbb{S}_{1}^{n+1}(C)$. If the mean curvature $\left|H^{2}\right|$ of $\left(M^{\prime}, g^{\prime}\right)$ satisfies the inequality

$$
\left|H^{2}\right|<C,
$$

then $\left(M^{\prime}, g^{\prime}\right)$ is a round $n$-sphere. Furthermore, if $\left(M^{\prime}, g^{\prime}\right)$ is simply-connected, then $\left(M^{\prime}, g^{\prime}\right)$ is isometric to a Euclidian sphere $\$^{n}$ of radius $\left(C-\left|H^{2}\right|\right)^{-1 / 2}$.

Montiel (see [8]) proved that every compact space-like surface in $\$_{1}^{n+1}(C)$ of constant mean curvature is totally umbilical. Based on these results and Corollary 2.4 , we can formulate the following obvious corollary. 
Corollary 2.4. Let $\left(M^{\prime}, g^{\prime}\right)$ be a compact simply-connected space-like hypersurface in a de Sitter space $\mathbb{\$}_{1}^{n+1}(C)$. If the mean curvature $\left|H^{2}\right|$ of $\left(M^{\prime}, g^{\prime}\right)$ is constant and

$$
\left|H^{2}\right|<C,
$$

then $\left(M^{\prime}, g^{\prime}\right)$ is isometric to a Euclidian sphere $\mathbb{\$}^{n}$ of radius $\left(C-\left|H^{2}\right|\right)^{-1 / 2}$.

\section{MAXIMAL SPACE-LIKE HYPERSURFACES IN LORENTZIAN MANIFOLDS}

Let $\left(M^{\prime}, g^{\prime}\right)$ be an $n$-dimensional complete space-like maximal hypersurface in a Lorentzian manifold $(M, g)$.

Theorem 3.1. Let $\left(M^{\prime}, g^{\prime}\right)$ be a space-like complete non-totally geodesic, maximal hypersurface in a Lorentzian manifold $(M, g)$. If there exists a positive constant $\delta$ such that the space-like Ricci curvature and time-like sectional curvature of $(M, g)$ satisfy the inequality

$$
\operatorname{Ric}\left(f_{*} V_{x}^{\prime}\right) \geq \sec \left(\mathcal{N}_{x} \wedge f_{*} V_{x}^{\prime}\right)+\delta
$$

for any vector $V_{x} \in T_{x} M^{\prime}$ at each point $x \in M^{\prime}$, then $\left(M^{\prime}, g^{\prime}\right)$ is compact, its diameter satisfies the inequality

$$
\operatorname{diam} M^{\prime} \leq \frac{\pi}{\sqrt{\delta}}
$$

and its first fundamental group is finite.

Proof. Firstly, from (1.8), we obtain

$$
\begin{aligned}
\operatorname{Ric}^{\prime}\left(V_{x}^{\prime}\right) & =\operatorname{Ric}\left(f_{*} V_{x}^{\prime}\right)-\sec \left(\mathcal{N}_{x} \wedge f_{*} V_{x}^{\prime}\right)+\left\|\mathcal{A} V^{\prime}\right\|^{2} \cdot\left\|V_{x}^{\prime}\right\|^{-2} \\
& \geq \operatorname{Ric}\left(V_{x}^{\prime}\right)-\sec \left(\mathcal{N}_{x} \wedge f_{*} V_{x}^{\prime}\right)
\end{aligned}
$$

for the nonzero vector $V_{x} \in T_{x} M^{\prime}$. Secondly, if we suppose that

$$
\operatorname{Ric}\left(f_{*} V_{x}^{\prime}\right)-\sec \left(\mathcal{N}_{x} \wedge f_{*} V_{x}^{\prime}\right) \geq \delta>0
$$

for some positive constant $\delta$ and an arbitrary nonzero vector $V_{x} \in T_{x} M^{\prime}$ at each point $x \in M^{\prime}$, then $\operatorname{Ric}^{\prime}\left(V_{x}^{\prime}\right) \geq \delta>0$. This means that $\left(M^{\prime}, g^{\prime}\right)$ is compact, its diameter satisfies the inequality

$$
\operatorname{diam} M^{\prime} \leq \frac{\pi}{\sqrt{\delta}}
$$

and the first fundamental group is finite (see [5, p. 216]). In particular, if we suppose for $(M, g)$ that its all time-like sectional curvatures $\sec (x, \pi) \leq-\delta<0$ and spacelike Ricci curvatures $\operatorname{Ric}(V) \geq 0$ for all space-like nonzero vectors $V$, then (1.8) implies that

$$
\operatorname{Ric}^{\prime}\left(V_{x}^{\prime}\right)=\operatorname{Ric}\left(f_{*} V_{x}^{\prime}\right)-\sec \left(\mathcal{N}_{x} \wedge f_{*} V_{x}^{\prime}\right)+\left\|\mathcal{A} V^{\prime}\right\|^{2} \cdot\left\|V_{x}^{\prime}\right\|^{-2} \geq \delta>0
$$

and hence, $\left(M^{\prime}, g^{\prime}\right)$ is compact. This completes the proof. 
Next, from (1.9), we obtain

$$
s^{\prime}=s+2 \operatorname{Ric}(\mathcal{N}, \mathcal{N})+\sum_{i=1, \ldots, n}\left(\lambda_{i}\right)^{2},
$$

where $\lambda_{1}, \ldots, \lambda_{n}$ are eigenvalues of $\mathcal{A}$ which are called the principal curvatures of $\left(M^{\prime}, g^{\prime}\right)$. In addition, we recall that $(M, g)$ is said to satisfy the strong energy condition or the time-like convergence condition (see [6, p. 95]) if $\operatorname{Ric}\left(V_{x}, V_{x}\right) \geq 0$ for every time-like vector $V_{x} \in T_{x} M$ at each point $x \in M$. Then, the following corollary holds.

Corollary 3.1. Let $\left(M^{\prime}, g^{\prime}\right)$ be a space-like and maximal hypersurface Lorentzian manifold $(M, g)$ which satisfies the strong energy condition, then $s^{\prime} \geq s$ for the scalar curvatures $s^{\prime}$ and $s$ of $\left(M^{\prime}, g^{\prime}\right)$ and $(M, g)$, respectively. The equality holds on a totally geodesic hypersurface.

Cheng and Yau [3] and T. Ishihara [7] proved that a complete maximal space-like submanifold $\left(M^{\prime}, g^{\prime}\right)$ of $\mathbb{S}_{1}^{n+1}(C)$ is totally geodesic. Then, the Ricci curvature Ric' of a space-like hypersurface $\left(M^{\prime}, g^{\prime}\right)$ in $\mathbb{S}_{1}^{n+1}(C)$ has the form

$$
\operatorname{Ric}^{\prime}\left(X^{\prime}\right)=(n-1) C>0
$$

for any nonzero $X^{\prime} \in T M^{\prime}$. This inequality is a necessary condition for a complete Riemannian manifold $\left(M^{\prime}, g^{\prime}\right)$ to be compact (see [5, p. 216]). Thus, using this fact and Mayer's theorem (see [5, pp. 212-213]), we can formulate the following corollary.

Corollary 3.2. If $\left(M^{\prime}, g^{\prime}\right)$ is a space-like complete maximal hypersurface in a de Sitter space-time $\mathbb{S}_{1}^{n+1}(C)$, then $\left(M^{\prime}, g^{\prime}\right)$ is compact, totally geodesic and its first fundamental group is finite. Further suppose that $\left(M^{\prime}, g^{\prime}\right)$ is simply-connected then $\left(M^{\prime}, g^{\prime}\right)$ is isometric to a Euclidian sphere $\mathbb{S}^{n}$ of radius $C^{-1 / 2}$.

\section{REFERENCES}

[1] J. A. Aledo and L. J. Alías, "On the volume and the Gauss map image of spacelike hypersurfaces in de Sitter space," Proc. Am. Math. Soc., vol. 130, pp. 1145-1151, 2002.

[2] J. K. Beem and P. E. Ehrlich, Global Lorentzian Geometry. New York: Marcel Dekker, 1981.

[3] S. Y. Cheng and S. T. Yau, "Maximal space-like hypersurfaces in the Lorentz-Minkowski spaces," Ann. Math., vol. 104, pp. 407-419, 1976.

[4] L. P. Eisenhart, Riemannian geometry. Princeton: University Press, 1926.

[5] D. Gromol, W. Klingenberg, and W. Meyer, Riemannsche geometrie im grossen. BerlinHeidelberg-New York: Springer-Verlag, 1975.

[6] S. W. Hawking and G. F. R. Ellis, The large scale structure of space-time. Cambridge University Press, 1973.

[7] T. Ishihara, "Maximal spacelike submanifolds of a pseudo-Riemannian space of constant curvature," Mich. Math. J., vol. 35, pp. 345-352, 1988.

[8] S. Montiel, "An integral inequality for compact spacelike hypersurfaces in de Sitter space and applications to the case of constant mean curvature," Indiana Univ. Math. J., vol. 37, pp. 909-917, 1988. 
Authors' addresses

Irina I. Tsyganok

Finance University under the Government of Russian Federation, Department of Probability Theory and Mathematical Statistics, 49-55 Leningradski Ave., 125468 Moscow, Russia

E-mail address: i.i.tsyganok@mail.ru

Sergey E. Stepanov

Finance University under the Government of Russian Federation, Department of Mathematics, 49-55 Leningradski Ave., 125468 Moscow, Russia

E-mail address: s.e.stepanov@mail.ru 